\title{
EVALUACIÓN DEL COLOR EN PULPA DE TOMATES DESHIDRATADOS DULCES PARA LA ELABORACIÓN DE CONFITURAS ${ }^{1}$
}

\author{
Delia Paola Urfalino-Rodríguez $z^{2}$, Jesica Worlock-Hughes ${ }^{2}$
}

\begin{abstract}
RESUMEN
Evaluación del color en pulpa de tomates deshidratados dulces para la elaboración de confituras. El objetivo del presente estudio fue evaluar el color de tomates deshidratados dulces, para la elaboración de confituras. El estudio se realizó durante 2013 en el Laboratorio de Deshidratado de Frutas y Hortalizas perteneciente al Instituto Nacional de Tecnología Agropecuaria (INTA), Estación Experimental Agropecuaria Rama Caída, Argentina. Se utilizaron tomates variedad Franco; estos se pelaron y cortaron, luego se formaron ocho grupos de $0,5 \mathrm{~kg}$ cada uno para realizar cuatro tratamientos con dos repeticiones. Los tomates se cubrieron con sacarosa $(0,5 \mathrm{~kg})$ mezclada con cuatro dosis de metabisulfito de sodio A: $0 \%$; B: $0,2 \%$; C: $0,5 \%$; D: $0,8 \%$, y se dejaron en reposo durante veinticuatro horas. Se enjuagaron y se deshidrataron en horno eléctrico con ventilación forzada a $55{ }^{\circ} \mathrm{C}$ hasta alcanzar una actividad acuosa de 0,47 $\pm 0,02$ (humedad inferior a 20\%). Posteriormente, se midió cada tres meses, el color en la pulpa de los tomates deshidratados y se determinó el contenido de dióxido de azufre residual al transcurrir un año de almacenamiento. Los cuatro tratamientos evaluados presentaron colores estables en el tiempo; sin embargo, los tratamientos $\mathrm{C}$ y $\mathrm{D}$ fueron los que obtuvieron mayores valores en el espacio de color CIE L*, $\mathrm{a}^{*}, \mathrm{~b}^{*}$ (colores rojos más intensos y atractivos). A su vez, dichos tratamientos fueron los que mayor cantidad de SO2 residual contenían, pero se encontraban por debajo del límite permitido por el Código Alimentario Argentino para la elaboración de confituras (C $160 \pm 22,63$; D 1,160 $\pm 214,96$ ppm).
\end{abstract}

\begin{abstract}
Pulp colour evaluation of dried sweet tomatoes to prepare jams. The objective of this study was to evaluate the colour of dried sweet tomatoes, to prepare jams. The assay was carried out during 2013 at the Fruits and Vegetables Drying Laboratory from the National Institute of Agricultural Technology (INTA) Rama Caída Agricultural Experimental Station, Argentina. Franco variety tomatoes were used; these were peeled and cut, then 8 groups of 0,5 $\mathrm{kg}$ each were formed in order to attain 4 treatments with two repetitions. The tomatoes were covered with sucrose $(0,5 \mathrm{~kg})$ mixed with four doses of sodium metabisulfite (A: $0 \%$; B: $0,2 \%$; C: $0,5 \%$; D: $0,8 \%$ ) and were left resting 24 hours. They were rinsed and dehydrated in an electric oven with forced ventilation at $55{ }^{\circ} \mathrm{C}$ until $0,47 \pm 0,02$ (humidity inferior to $20 \%$ ) of water activity was reached. Subsequently, the colour of the pulp of the dried tomatoes was measured every three months and the content of sulphur dioxide was determined after one year in storage. The four treatments evaluated presented stable colours in time; however, treatments $\mathrm{C}$ and $\mathrm{D}$ showed the highest values in colour space CIE $\mathrm{L}^{*}, \mathrm{a}^{*}, \mathrm{~b}^{*}$ (most intense and attractive red colours). Also, these treatments were the ones with the greatest residual amount of sulphur dioxide, however, they were below of the allowed limit established by the Argentine Food Code for the manufacturing of jams(C $160 \pm 22,63$; D $1.160 \pm 214,96 \mathrm{ppm})$.
\end{abstract}

Keywords: Solanum lycopersicon Mill., osmoticconvective drying, tomato jam.

Palabras clave: Solanum lycopersicon Mill., deshidratación osmótica-convectiva, confitura de tomate.

\footnotetext{
1 Recibido: 17 de junio, 2015. Aceptado: 24 de julio, 2015. El presente trabajo forma parte del Módulo Tecnología de Transformación de Productos Frutihortícolas perteneciente al proyecto específico: Tecnologías de transformación de alimentos. Programa Nacional - Agroindustria y Valor Agregado en Origen (PNAIYAV 1130032). Mendoza, Argentina.

2 Instituto Nacional de Tecnología Agropecuaria, Estación Experimental Agropecuaria, Rama Caída, El Vivero s/n Rama Caída, San Rafael, Mendoza, Argentina. C.P.: 5603.urfalino.delia@inta.gob.ar (autor para correspondencia), worlock.jesica@inta.gob.ar
} 


\section{INTRODUCCIÓN}

La producción de tomate presenta cierta estacionalidad anual que depende de las características agroclimáticas de la región de cultivo. Sin embargo, el desarrollo tecnológico ha permitido superar esta limitación a través del procesamiento de los frutos. Las industrias elaboran y almacenan productos derivados del tomate con los cuales pueden satisfacer la demanda de los consumidores durante todo el año (IDR, 2014).

La diversificación de estos productos ofrece distintas opciones adecuadas para cada necesidad; entre estas se encuentran los tomates deshidratados dulces, que son un producto intermedio estable, apto para la elaboración de una gran variedad de confituras ${ }^{3}$.

La deshidratación es uno de los métodos más antiguos de conservación de alimentos conocido por el hombre. El proceso involucra la remoción de la mayor parte del agua del alimento para evitar la actividad enzimática y el desarrollo de microorganismos (Rahman, 2003).

El deshidratado convencional de tomates ocasiona efectos adversos en la calidad final del producto. El tejido de la fruta se oscurece durante el secado y se desarrolla un fuerte sabor característico (Gupta y Nath, 1984 ). El color de un producto tiene una gran influencia en la percepción de calidad y aceptación por parte del consumidor. Durante el procesamiento térmico se presentan muchas reacciones que afectan este atributo, entre ellas las más comunes son degradación de los pigmentos, reacciones de pardeamiento (enzimático y no enzimático) y oxidación del ácido ascórbico. Otros factores que afectan el color pueden ser $\mathrm{pH}$ del fruto, acidez, temperatura y duración del procesamiento, cultivar y contaminación con metales pesados (Maskan, 2001).

La temperatura ideal para efectuar la deshidratación de tomates es entre 45 y $55^{\circ} \mathrm{C}$, lo cual permite que el producto retenga sus nutrientes (incluyendo vitaminas y licopeno, el nutriente responsable del color rojo intenso de los tomates), aromas y sabores (Andritsos et al., 2003).

\footnotetext{
Las confituras de tomate son productos obtenidos mediante la cocción de los frutos, sus jugos y/o pulpas, con el agregado de azúcares, que pueden ser reemplazados parcial o totalmente por miel (ANMAT, Art. 807 - Dec. 112, 12.1.76). Ej.: compotas, frutas en almíbar, mermeladas, dulces, jaleas, dulces mixtos, frutas confitadas, etc.
}

El oscurecimiento o pardeamiento de los tejidos del tomate es un fenómeno natural que puede disminuirse dramáticamente con el uso de azufre. La adición de este elemento, previo al proceso de secado, inhibe los pardeamientos enzimáticos (polifenoloxidasa) y no enzimáticos (Reacción de Maillard), ejerce una acción antioxidante (elimina el oxígeno y actúa como agente reductor), inhibe ciertas enzimas como proteasas, oxidasas y peroxidasas, es antimicrobiano y fungistático y, a su vez, facilita la deshidratación por plasmólisis de células (Stafford y Bolin,1972; Ledbetter et al., 2002; Doymaz, 2004; Latapi y Barrett, 2006).

Para minimizar el deterioro del color durante la deshidratación de tomates, se utilizan métodos combinados de tratamiento como por ejemplo la deshidratación osmótica y la aplicación de metabisulfito de sodio. La deshidratación osmótica aplicada como pre-tratamiento en los procesos tradicionales de conservación, ha cobrado gran interés debido a que mejora la calidad organoléptica del producto final con un bajo consumo de energía (Huayamave y Cornejo, 2005); durante este proceso, el tomate se coloca en una solución hipertónica (concentrada), en la cual se llevan a cabo dos flujos de transferencia de masa en contracorriente, a saber, un flujo de agua desde el tomate a la solución y una migración de solutos de esta al tomate. Los solutos más empleados para efectuar tratamientos osmóticos son azúcares y sales, principalmente sacarosa y cloruro de sodio (Tonon et al., 2007).

El objetivo del presente estudio fue evaluar el color de tomates deshidratados dulces, para la elaboración de confituras.

\section{MATERIALES Y MÉTODOS}

\section{Selección de material vegetal y su preparación}

El ensayo se llevó a cabo en el Laboratorio de Deshidratado de Frutas y Hortalizas, perteneciente a la Estación Experimental Agropecuaria Rama Caída, durante el año 2013. Se utilizaron tomates (Solanum lycopersicon Mill. variedad Franco) frescos, sanos, limpios y maduros (color rojo intenso uniforme).

Los tomates se colocaron en bolsas plásticas de red y se sumergieron durante veinte segundos en agua en ebullición ( $98^{\circ} \mathrm{C}$ aproximadamente), para facilitar la remoción de la piel. Posteriormente, se pelaron manualmente, se cortaron en mitades longitudinales 
y se pesaron. Se formaron ocho grupos de tomates de $0,5 \mathrm{~kg}$ cada uno para realizar cuatro tratamientos con dos repeticiones, en un diseño experimental completamente aleatorizado y evaluando los datos a través de un análisis de la varianza.

\section{Tratamientos efectuados}

Los tomates se cubrieron con sucesivas capas de sacarosa (azúcar común de mesa Tipo A), empleando un total de $0,5 \mathrm{~kg}$. Dependiendo del tratamiento, se adicionaron distintas dosis de metabisulfito de sodio $\left(\mathrm{Na}_{2} \mathrm{~S}_{2} \mathrm{O}_{5}, \mathrm{E} 223\right)$ en polvo mezclado con la sacarosa para ejercer acción conservante y antioxidante. Los tomates permanecieron en esta etapa (deshidratado osmótico) durante veinticuatro horas. Posteriormente, los mismos se enjuagaron rápidamente con agua potable y se sometieron a una deshidratación en un horno eléctrico con ventilación forzada a $55{ }^{\circ} \mathrm{C}$. La deshidratación finalizó cuando los frutos alcanzaron una actividad acuosa de 0,47 $\pm 0,02$ (humedad inferior a 20\% ${ }^{4}$ ).

A continuación se detallan los tratamientos efectuados:

Tratamiento (T) A: $0,5 \mathrm{~kg}$ de tomates con $0,5 \mathrm{~kg}$ de sacarosa.

Tratamiento (T) B: $0,5 \mathrm{~kg}$ de tomates con $0,5 \mathrm{~kg}$ de sacarosa y $2 \mathrm{~g} \mathrm{Na}_{2} \mathrm{~S}_{2} \mathrm{O}_{5}(0,2 \%)$.

Tratamiento (T) C: $0,5 \mathrm{~kg}$ de tomates con $0,5 \mathrm{~kg}$ de sacarosa y $5 \mathrm{~g} \mathrm{Na}_{2} \mathrm{~S}_{2} \mathrm{O}_{5}(0,5 \%)$.

Tratamiento (T) D: $0,5 \mathrm{~kg}$ de tomates con $0,5 \mathrm{~kg}$ de sacarosa y $8 \mathrm{~g} \mathrm{Na}_{2} \mathrm{~S}_{2} \mathrm{O}_{5}(0,8 \%)$.

\section{Determinaciones realizadas en los tomates deshidratados dulces}

\section{Color}

Se midió el color de la pulpa (lado convexo) de los tomates deshidratados dulces cada tres meses durante un año con un colorímetro Kónica-Minolta CR400. Las determinaciones se realizaron en un ambiente con

\footnotetext{
Según el Código Alimentario Argentino (Art. 904 bis Res. Conj. SPReI $N^{\circ} 169 / 2013$ y SAGyP $N^{\circ} 230 / 2013$ ) la fruta deshidratada no debe contener más de $25 \%$ de agua, si se coloca en envases herméticos se permite un máximo de $35 \%$. Sin embargo, para evitar cualquier tipo de inconvenientes durante el almacenamiento, es preferible que el porcentaje de humedad de los tomates deshidratados sea inferior a $20 \%$.
}

temperatura constante $\left(20^{\circ} \mathrm{C}\right)$. Se tomaron tres puntos distintos de medición de manera aleatoria por tomate.

El instrumento se calibró con un plato cerámico de color blanco. La escala de color utilizada fue CIE $\mathrm{L}^{*} \mathrm{a}^{*} \mathrm{~b}^{*}$ (CIELAB) la cual es uniforme y el espacio de color está organizado en forma de cubo. El valor máximo de $\mathrm{L}^{*}$ es 100 que representa una perfecta reflectancia difusa (blanco), y el valor mínimo es 0 el cual representa el negro. Los valores de $a^{*}$ y b* no tienen un límite numérico específico. Cuando $a^{*}$ es positiva representa el rojo y cuando es negativa el verde. Cuando $\mathrm{b}^{*}$ es positivo representa amarillo y cuando es negativo azul (HunterLab, 1996).

\section{Dióxido de azufre $\left(\mathrm{SO}_{2}\right)$ residual}

Las muestras se analizaron luego de transcurrir un año de almacenamiento de acuerdo con el método oficial de la AOAC (2006), 990.28 Monier-Williams optimizado.

Se colocaron $30 \mathrm{ml}$ de agua oxigenada diez volúmenes, tres gotas de rojo de metilo y tres gotas de hidróxido de sodio $0,1 \mathrm{~N}$ en una probeta de $100 \mathrm{ml}$. Se vertieron $400 \mathrm{ml}$ de agua destilada en un balón de tres bocas. Se armó el conjunto y se realizó un barrido con nitrógeno gaseoso a través de $500 \mathrm{ml}$ de carbonato de sodio al $10 \%$, durante cinco minutos. Se añadieron al balón 20 g de muestra y $90 \mathrm{ml}$ de ácido clorhídrico $4 \mathrm{~N}$, se tapó y se hizo hervir el conjunto durante 1'45" horas en corriente de $\mathrm{N}_{2}$. Se lavó la probeta y el refrigerante recogiendo el agua de lavado en matraz y se tituló con hidróxido de sodio $0,1 \mathrm{~N}$. La concentración de $\mathrm{SO}_{2}$, en ppm, se calculó según la ecuación:

$$
\mathrm{SO}_{2} \mathrm{ppm}=\frac{\mathrm{V} \times 32.03 \times 1000}{\mathrm{G}}
$$

Siendo:

$\mathrm{V}=\mathrm{ml}$ de hidróxido de sodio utilizado.

$\mathrm{G}=$ peso de muestra, en gramos.

$32,03=$ peso miliequivalente del $\mathrm{SO}_{2}$.

$1000=$ factor de conversión de miliequivalentes a microequivalentes.

\section{RESULTADOS Y DISCUSIÓN}

\section{Medición de color}

Para los tomates deshidratados dulces, el color rojo intenso acompañado por un aroma agradable, son 
indicadores de calidad; también son características deseadas para la elaboración de una gran variedad de confituras; sin embargo, durante el almacenamiento, los tomates deshidratados experimentan cambios de color, como consecuencia de la oxidación de los carotenoides (Ochoa-Reyes et al., 2013).

$\mathrm{L}^{*}$ es el parámetro de mayor importancia durante el periodo de almacenamiento. Cuando este disminuye, significa que el producto comienza a oscurecerse y, por lo tanto, a perder su atractivo.

En el primer mes no existieron diferencias estadísticamente significativas para el valor de $\mathrm{L}^{*}$ entre los cuatro tratamientos efectuados.

A partir del tercer mes y hasta el doceavo mes inclusive, el tratamiento A tuvo menores valores de $\mathrm{L}^{*}$ que los otros tres, con diferencias estadísticamente significativas. Este resultado indicó que los tomates pertenecientes al tratamiento A poseían un color más oscuro que los tomates de los otros tres; esto se debio a que el oscurecimiento o pardeamiento de los tejidos del tomate es un fenómeno natural, al no aplicarle metabisulfito de sodio previo al proceso de deshidratado, es esperable que $L^{*}$ disminuya más rápidamente con el transcurso del tiempo (Figura 1).

Para los tratamientos $\mathrm{B}, \mathrm{C}$ y D el parámetro $\mathrm{L}^{*}$ no presentó diferencias estadísticamente significativas durante todo el periodo evaluado.

El comportamiento del parámetro a* varió en el intervalo de tiempo evaluado.

En el primer mes no se observaron diferencias estadísticamente significativas entre los cuatro tratamientos efectuados.

Durante el tercer mes los tratamientos B y D no presentaron diferencias significativas mientras que los tratamientos A y C sí.

En el sexto mes no existieron diferencias estadísticamente significativas entre los cuatro tratamientos aplicados.

En el noveno mes, el tratamiento A presentó diferencias estadísticamente significativas y obtuvo el menor valor de $\mathrm{a}^{*}$; es decir, disminuyó su intensidad de color rojo. Los tratamientos B, C, y D no presentaron diferencias significativas entre sí.

Transcurrido un año de evaluación, los tratamientos C y D no presentaron diferencias estadísticamente significativas, ambos obtuvieron los mayores valores de $\mathrm{a}^{*}$; es decir, que fueron los que presentaron un color rojo más intenso transcurrido un año de

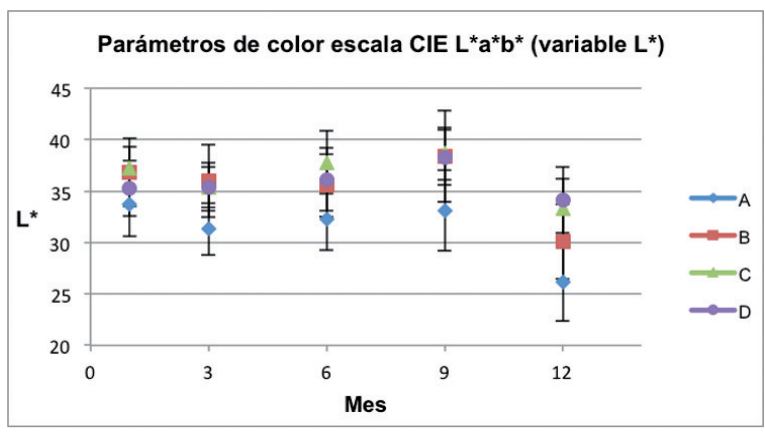

Figura 1. Evaluación del color en pulpa de tomates deshidratados dulces (Solanum lycopersicon Mill. variedad Franco) mediante un colorímetro KónicaMinolta CR 400. Se utilizó el espacio de color CIE L*a*b* (parámetro evaluado L*). Estación Experimental Agropecuaria Rama Caída, Argentina, durante el año 2013.

Tratamiento $0,5 \mathrm{~kg}$ de tomate con $\mathrm{A}=0,5 \mathrm{~kg}$ de sacarosa; $\mathrm{B}=0,5 \mathrm{~kg}$ de sacarosa y $2 \mathrm{~g}$ de $\mathrm{Na}_{2} \mathrm{~S}_{2} \mathrm{O}_{5}$ $(0,2 \%) ; \mathrm{C}=0,5 \mathrm{~kg}$ de sacarosa y $5 \mathrm{~g} \mathrm{Na}_{2} \mathrm{~S}_{2} \mathrm{O}_{5}(0,5 \%)$; $\mathrm{D}=0,5 \mathrm{~kg}$ de sacarosa y $8 \mathrm{~g} \mathrm{Na}_{2} \mathrm{~S}_{2} \mathrm{O}_{5}(0,8 \%)$.

Figure 1. Dried sweet tomatoes (Solanum lycopersicon Mill. Franco variety) pulp colour evaluation with a Konica-Minolta CR 400 colorimeter. CIE L*a*b* space colour was used ( $\mathrm{L}^{*}$ parameter evaluated). Rama Caída Agricultural Experimental Station, in 2013.

Treatment of $0,5 \mathrm{~kg}$ of tomatoes with $\mathrm{A}: 0,5 \mathrm{~kg}$ of sucrose, B: $0,5 \mathrm{~kg}$ of sucrose and $2 \mathrm{~g}$ of $\mathrm{Na}_{2} \mathrm{~S}_{2} \mathrm{O}_{5}$ $(0,2 \%) ; \mathrm{C}: 0,5 \mathrm{~kg}$ of sucrose and $5 \mathrm{~g} \mathrm{Na}_{2} \mathrm{~S}_{2} \mathrm{O}_{5}(0,5 \%)$; D: $0,5 \mathrm{~kg}$ of sucrose and $8 \mathrm{~g} \mathrm{Na}_{2} \mathrm{~S}_{2} \mathrm{O}_{5}(0,8 \%)$.

almacenamiento. Los tratamientos A y B presentaron diferencias estadísticamente significativas, siendo A el que obtuvo el menor valor para el parámetro a*; es decir, que era el tratamiento con el color más oscuro.

A mayor dosis de metabisulfito aplicado en cada tratamiento, mayor fue valor de $\mathrm{a}^{*}$ obtenido; es decir, los tomates poseían un color rojo más intenso (Figura 2). El coeficiente de correlación calculado entre la dosis de metabisulfito de sodio aplicada y el valor del parámetro a* fue de $\mathrm{r}=0,72$ (p-valor $<0,0001$ ).

Los valores que adoptó el parámetro $b^{*}$ se mantuvieron estables para los cuatro tratamientos evaluados durante un año (Figura 3).

Desde el primero hasta el doceavo mes inclusive, el tratamiento A obtuvo valores de $b^{*}$ menores que los otros tres, con diferencias estadísticamente significativas. Esto indicó que los tomates 


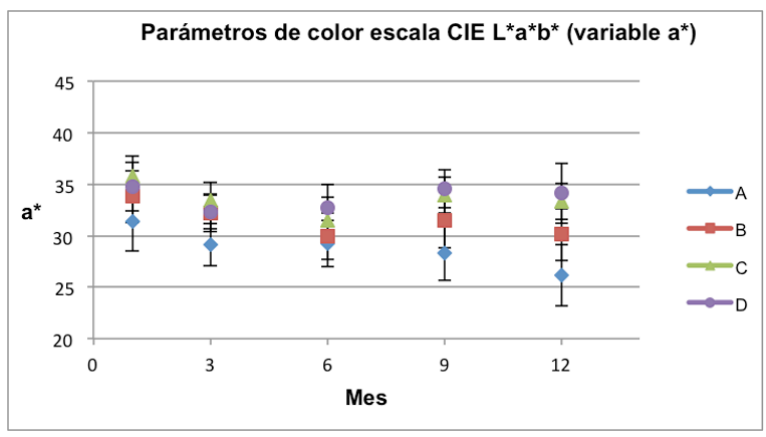

Figura 2. Evaluación del color en pulpa de tomates deshidratados dulces (Solanum lycopersicon Mill. variedad Franco) mediante un colorímetro KónicaMinolta CR 400. Se utilizó el espacio de color CIE L*a*b* (parámetro evaluado a*). Estación Experimental Agropecuaria Rama Caída, Argentina, durante el año 2013.

Tratamiento $0,5 \mathrm{~kg}$ de tomate con $\mathrm{A}=0,5 \mathrm{~kg}$ de sacarosa; $\mathrm{B}=0,5 \mathrm{~kg}$ de sacarosa y $2 \mathrm{~g}$ de $\mathrm{Na}_{2} \mathrm{~S}_{2} \mathrm{O}_{5}$ $(0,2 \%) ; \mathrm{C}=0,5 \mathrm{~kg}$ de sacarosa y $5 \mathrm{~g} \mathrm{Na}_{2} \mathrm{~S}_{2} \mathrm{O}_{5}(0,5 \%)$; $\mathrm{D}=0,5 \mathrm{~kg}$ de sacarosa y $8 \mathrm{~g} \mathrm{Na} \mathrm{S}_{2} \mathrm{O}_{5}(0,8 \%)$.

Figure 2. Dried sweet tomatoes (Solanum lycopersicon Mill. Franco variety) pulp colour evaluation with a KonicaMinolta CR 400 colorimeter. CIE L*a*b* space colour was used (a* parameter evaluated). Rama Caída Agricultural Experimental Station, in 2013.

Treatment of $0,5 \mathrm{~kg}$ of tomatoes with A: $0,5 \mathrm{~kg}$ of sucrose, B: $0,5 \mathrm{~kg}$ of sucrose and $2 \mathrm{~g}$ of $\mathrm{Na}_{2} \mathrm{~S}_{2} \mathrm{O}_{5}$ $(0,2 \%) ; \mathrm{C}: 0,5 \mathrm{~kg}$ of sucrose and $5 \mathrm{~g} \mathrm{Na}_{2} \mathrm{~S}_{2} \mathrm{O}_{5}(0,5 \%)$; D: $0,5 \mathrm{~kg}$ of sucrose and $8 \mathrm{~g} \mathrm{Na}_{2} \mathrm{~S}_{2} \mathrm{O}_{5}(0,8 \%)$.

pertenecientes a este tratamiento eran más oscuros que los tomates de los otros tratamientos.

En los tratamientos B, C y D el parámetro b* no presentó diferencias significativas en todo el período de tiempo evaluado.

Los cuatro tratamientos evaluados resultaron satisfactorios, presentaron un color estable en el tiempo (Figura 4); sin embargo, los tratamientos $\mathrm{C} \mathrm{y}$ $\mathrm{D}$ fueron los que presentaron mayores valores de $\mathrm{L}^{*}$, $a^{*}$ y $b^{*}$ obtuvieron; es decir, que estos presentaron colores rojos más intensos y atractivos durante el período de un año, ideales para la elaboración de confituras de excelente calidad.

\section{Dióxido de azufre $\left(\mathrm{SO}_{2}\right)$ residual}

La determinación del contenido de dióxido de azufre residual se efectuó exclusivamente en los tratamientos B, C y D, a los cuales se les aplicó metabisulfito de sodio $\left(\mathrm{Na}_{2} \mathrm{~S}_{2} \mathrm{O}_{5}\right)$.

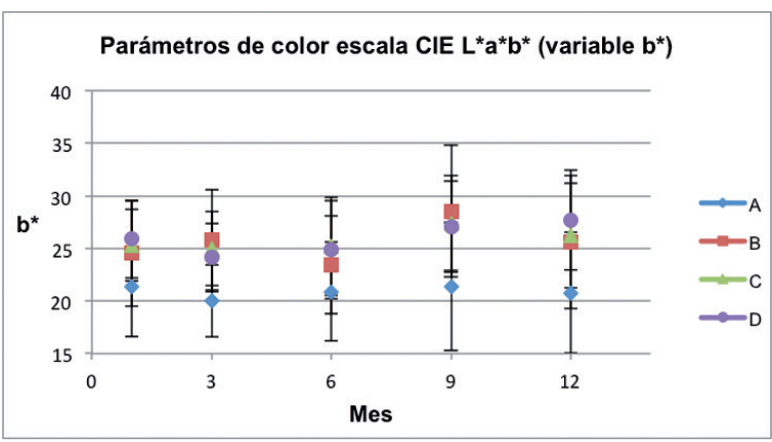

Figura 3. Evaluación del color en pulpa de tomates deshidratados dulces (Solanum lycopersicon Mill. variedad Franco) mediante un colorímetro KónicaMinolta CR 400. Se utilizó el espacio de color CIE L*a*b* (parámetro evaluado b*). Estación Experimental Agropecuaria Rama Caída, Argentina, durante el año 2013.

Tratamiento $0,5 \mathrm{~kg}$ de tomate con $\mathrm{A}=0,5 \mathrm{~kg}$ de sacarosa; $\mathrm{B}=0,5 \mathrm{~kg}$ de sacarosa y $2 \mathrm{~g}$ de $\mathrm{Na}_{2} \mathrm{~S}_{2} \mathrm{O}_{5}$ $(0,2 \%) ; \mathrm{C}=0,5 \mathrm{~kg}$ de sacarosa y $5 \mathrm{~g} \mathrm{Na}_{2} \mathrm{~S}_{2} \mathrm{O}_{5}(0,5 \%)$; $\mathrm{D}=0,5 \mathrm{~kg}$ de sacarosa y $8 \mathrm{~g} \mathrm{Na}_{2} \mathrm{~S}_{2} \mathrm{O}_{5}(0,8 \%)$.

Figure 3. Dried sweet tomatoes (Solanum lycopersicon Mill. Franco variety) pulp colour evaluation of with a Konica-Minolta CR 400 colorimeter. CIE $\mathrm{L} * \mathrm{a} * \mathrm{~b} *$ space colour was used $(\mathrm{b} *$ parameter evaluated). Rama Caída Agricultural Experimental Station, in 2013.

Treatment of $0,5 \mathrm{~kg}$ of tomatoes with A: 0,5 $\mathrm{kg}$ of sucrose, B: $0,5 \mathrm{~kg}$ of sucrose and $2 \mathrm{~g}$ of $\mathrm{Na}_{2} \mathrm{~S}_{2} \mathrm{O}_{5}(0,2 \%)$; C: $0,5 \mathrm{~kg}$ of sucrose and $5 \mathrm{~g}$ $\mathrm{Na}_{2} \mathrm{~S}_{2} \mathrm{O}_{5}(0,5 \%)$; D: $0,5 \mathrm{~kg}$ of sucrose and $8 \mathrm{~g}$ $\mathrm{Na}_{2} \mathrm{~S}_{2} \mathrm{O}_{5}(0,8 \%)$.

En el tratamiento B no se detectó dióxido de azufre residual a través de la técnica Williams-Monier, a pesar de que se le adicionó $0,2 \%$ de $\mathrm{Na}_{2} \mathrm{~S}_{2} \mathrm{O}_{5}$. Es probable que el mismo se haya ido perdiendo durante el transcurso del año; esto coincide con lo observado por otros investigadores que encontraron pérdidas significativas en el contenido de $\mathrm{SO}_{2}$ en tomates sulfitados y deshidratados al sol luego de tres meses de almacenamiento (Latapi y Barrett, 2006).

$\mathrm{Al}$ tratamiento $\mathrm{C}$ se le agregó $0,5 \%$ de $\mathrm{Na}_{2} \mathrm{~S}_{2} \mathrm{O}_{5}, \mathrm{y}$ con esa dosis se detectaron $160 \mathrm{ppm} \pm 22,63$.

Los tomates deshidratados dulces correspondientes al tratamiento $\mathrm{D}$ presentaron un contenido de dióxido azufre residual de $1160 \mathrm{ppm} \pm 214,96$ (Cuadro 1). Este producto se consideraría inadecuado para el consumo directo como alimento deshidratado. El Código Alimentario Argentino (Art. 916 - Res. Conj. SPReI No 169/2013 y SAGyP N²30/2013) 


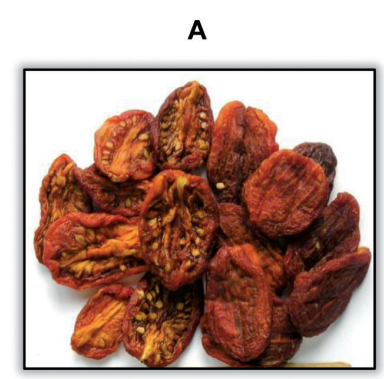

C

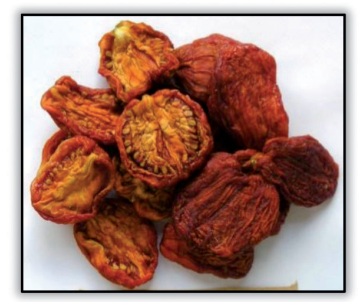

B

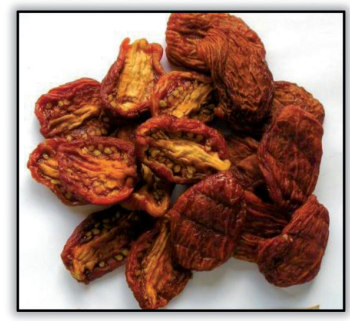

D

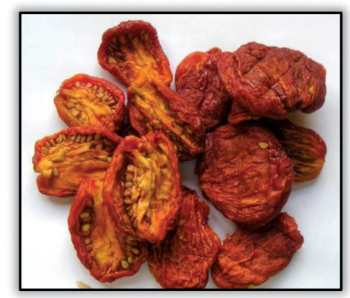

Figura 4. Tomates deshidratados dulces (Solanum lycopersicon Mill. variedad Franco) para la elaboración de confituras, transcurrido un año de almacenamiento. Estación Experimental Agropecuaria Rama Caída, Argentina, en el año 2014.

Figure 4. Dried sweet tomatoes (Solanum lycopersicon Mill. Franco variety) to prepare jams after one year of storage. Rama Caída Agricultural Experimental Station, Argentina. 2014.

(Ministerio de Salud - Presidencia de la Nación, 1969), establece un máximo de $\mathrm{SO}_{2}$ de 1000 ppm para productos deshidratados. Sin embargo, como se trata de un producto intermedio, sujeto a una posterior elaboración de confituras, se permiten hasta $2500 \mathrm{ppm}$. Una vez obtenida la confitura, el producto terminado podrá contener como máximo 100 ppm de anhídrido sulfuroso total (Art. 807 - Dec. 112, 12.1.76) (Ministerio de Salud - Presidencia de la Nación, 1971).

De lo anteriormente expuesto se observa que los tratamientos $\mathrm{C}$ y $\mathrm{D}$ presentaron valores de dióxido de azufre residual dentro de los límites establecidos por el Código Alimentario Argentino para la elaboración de confituras.

El oscurecimiento o pardeamiento de los tejidos del tomate durante el proceso de deshidratación, es un fenómeno natural que afecta la percepción de calidad por parte de los consumidores. Su efecto puede disminuirse empleando una estrategia de deshidratado mediante la combinación de métodos, como la deshidratación osmótica junto con la adición de metabisulfito de sodio.
Cuadro 1. Determinación del contenido de dióxido de azufre $\left(\mathrm{SO}_{2}\right)$ residual en tomates deshidratados dulces (Solanum lycopersicon Mill. Variedad Franco) mediante el método oficial de la AOAC (2006) 990.28 Monier Williams (optimizado). Estación Experimental Agropecuaria Rama Caída, Argentina. 2014

Table 1. Determination of residual sulphur dioxide $\left(\mathrm{SO}_{2}\right)$ content in dried sweet tomatoes (Solanum lycopersicon Mill. Franco variety) with the AOAC official method (2006) 990.28 Monier Williams (optimized). Rama Caída Agricultural Experimental Station, Argentina. 2014.

\begin{tabular}{lcc}
\hline Tratamiento & $\begin{array}{c}\text { SO } \\
\text { (ppm) }\end{array}$ & $\begin{array}{c}\text { Prom. y desv. } \\
\text { Estándar }\end{array}$ \\
\hline A R1 & 0 & 0 \\
A R2 & 0 & 0 \\
B R1 & 0 & \\
B R2 & 0 & $160 \pm 22,63$ \\
C R1 & 176 & \\
C R2 & 144 & $1,160 \pm 214,96$ \\
D R1 & 1,008 & \\
D R2 & 1,312 & \\
\hline
\end{tabular}

\section{LITERATURA CITADA}

Andritsos, N., P. Dalampakis, and N. Kolios. 2003. Use of geothermal energy for tomato drying. GHC Bulletin, 24(1):9-13.

AOAC (Association of Official Analytical Chemistry) 2006. In Official methods of analysis of the association analytical chemistry. $18^{\text {th }}$ ed. AOAC International. WA, USA.

Doymaz, I. 2004. Effect of pre-treatments using potassium metabisulphide and alkaline ethyloleate on the drying kinetics of apricots. Biosyst. Eng. 89:281-287.

Gupta, R.G., and N. Nath. 1984. Drying of tomatoes. J. Food Sci. Tecnol. 21:372-376.

Huayamave, E.C., y Z.F. Cornejo. 2005. Influencia de presiones de vacío en la transferencia de masa durante la deshidratación osmótica del mango. Rev. Tecnol. ESPOL 18:141-145.

HunterLab. 1996. Hunter Lab Color Scale. Insight on Color 89 (August 1-15, 1996). Hunter Associates Laboratories. Reston, VA, USA.

IDR (Instituto de Desarrollo Rural). 2014. Red provincial de precios pagados a productor informes por producto 
tomate Mendoza - Periodo 1998-99 a 2012/2013

Informe de Avance 2014. http://www.idr.org.ar/ wp-content/uploads/2012/08/InformeTomate_ hasta2012-13_docx.pdf (consultado 23 jul. 2015).

Latapi, G., and D.M. Barrett. 2006. Influence of predrying treatments on quality and safety of sundried tomatoes. Part I: Use of Steam Blanching, Boiling Brine Blanching, and Dips in Salt or Sodium Metabisulfite. J. Food Sci. 71: S24-S31 DOI: 10.1111/ j.1365-2621.2006.tb12409.x

Ledbetter, C.A., L.H. Aung, and D.E. Palmquist. 2002. The effect of fruit maturity on quality and color shift of dried 'Patterson' apricot during eight months of coldstorage. J. Horticult. Sci. Biotechnol. 77:526-533.

Maskan, M. 2001. Kinetics of color change of kiwi fruits during hot air and microwave drying. J. Food Eng. 48:169-175.

Ministerio de la Salud - Presidencia de la Nación. 1969. Ley 18284, Código Alimentario Argentino, Boletín Oficial de la República Argentina. ARG.
Ministerio de la Salud - Presidencia de la Nación. 1971. Decreto $N^{\circ}$ 2126/71, Reglamento de la Ley 18284 , Boletín Oficial de la República Argentina. ARG.

Ochoa-Reyes, E., J.J. Ornelas-Paz, S. Ruiz-Cruz, V. IbarraJunquera, J.D. Pérez-Martínez, J.C. Guevara-Arauza, y C.N. Aguilar. 2013. Tecnologías de deshidratación para la preservación de tomate (Lycopersicon esculentum Mill.). Biotecnia XV (2):39-46.

Rahman, M.S. 2003. Manual de conservación de los alimentos. Editorial Acribia S.A., Zaragosa, ESP.

Stafford, A.E., and H.R. Bolin. 1972. Absorption of aqueous bisulfite by apricots. J. Food Sci. 37:941-943.

Tonon, R.V.,A.F. Baroni, and M.D. Hubinger. 2007. Osmotic dehydration of tomato in ternary solutions: Influence of process variables on mass transfer kinetics and an evaluation of the retention of carotenoids. J. Food Eng. 82:509-517. 
